\title{
Diabetes Mellitus and Associated Risk Factors among Tuberculosis Patients in Rawalpindi, Pakistan
}

\author{
Noureen* \\ Master degrees in Public Health and Political Science, Quiad-e-Azam University, Pakistan
}

Submission: February 23, 2017; Published: March 08, 2017

*Corresponding author: Noureen, Master degrees in Public Health and Political Science, Quaid-e-Azam University, Islamabad, Pakistan, Email: alshifanoor@yahoo.in

Abstract

Background: Tuberculosis (TB) and Diabetes Mellitus (DM) both diseases are highly prevalent in Pakistan. The coexistence of dual diseases has serious implication for each other that is neglected by the clinicians. The country has a high burden of TB along with huge DM prevalence. TB is one of the most common infectious diseases worldwide and the second leading cause of death as well. The community of the researchers had been working since several decades for an effective preventive strategy for TB. Although current preventive strategy against the TB had truly lowered its incidence but it is clear now that the problem is far from over. Therefore, the focus of researches now has been shifted to the previous untargeted risk factors that involved in the spread of TB. DM is one such factor that is well recognized now as an important risk factor for TB. Both diseases often exist together and complicate each other on many levels. A huge prevalence of DM may contribute the high prevalence of TB showed by the evidences of several researches. Now the experts are raising concerns for dual diseases as merging epidemics especially in low income and developing countries. As these countries have huge burden of TB with a fastest increase in the prevalence of Diabetes fueled by obesity. The quality of Diabetes treatment with disparate range makes the disease fueling the TB incidence in Pakistan. For the control and care for both diseases a collaborative framework by World Health Organization (WHO) and International Union against TB \& Lung diseases emphasized on bi-directional screening. But the impact of both diseases is still unappreciated in Pakistan.

Objective: To determine the Diabetes prevalence and associated risk factors among TB patients currently on treatment at a tertiary care hospital in Punjab, Pakistan.

Methodology: A cross-sectional hospital based study was utilized. Physician diagnosed 248 TB patients were selected by systematic sampling with a random start technique during the study period (March 2016 to June 2016). A pretested structured questionnaire was used to collect the data. Fasting glucose levels were carried out by finger puncture. Diagnosis of the DM was based on the WHO criteria. The details of socio demographic, treatment regimen and habitual risk factors were collected after the frequent visits to the Hospital. All the selected patients were undergoing anthropometric measurements by a well-trained investigator. SPSS software version 21 was used for statistical analysis. For testing the significance, categorical data was compared using a chi-square test while Logistic Regression technique was used to assess the effect of different risk factors of Diabetes among TB patients.

Results: Out of 248 patients $51 \%$ were males and $49 \%$ were females. Their age range was from 18 to 80 years with mean age of 42 years. Diabetes was $21 \%$ prevalent in TB patients which are more than expected. The study revealed that $14(7.28 \%)$ were newly diagnosed and the co-infected patients were in their productive ages. Weight loss and malnutrition was the predominant symptom in $91 \%$ patients. Majority patients were belonged to rural areas with monthly income less than ten thousand.

Conclusion: The study provided a re-echo need to raise the awareness of rapid increase of Diabetes with TB, yet a clinical importance and potential public health threat seems to be ignored largely in Pakistan. Robust action plans with quality implementation is urgently needed to address this dual disease burden. To address both diseases appropriate strategies are needed to manage the drastic situation with crossscreening patients for each disease. Irrespective of its causal or co-morbid factors good quality implementation is urgently needed for robust action plans to address this dual burden in Pakistan.

Keywords: Epidemic; Co-infection; Incidence; Biological Plausibility; Obesity; Chronic disease; Causal link; Meta-analytic Techniques Abbreviations: TB: Tuberculosis; DM: Diabetes Mellitus; WHO: World Health Organization; WC: Waist Circumference; SPSS: Software statistical Package for Social Sciences 


\section{Introduction}

In the early 20th century it was said that the patients who did not die in a diabetic coma were likely to do so from TB, particularly if they were poor. Roots in reviewing the whole history of dual diseases DM was like a death sentence within a period of five years and the ultimate death cause was TB before the insulin discovery.

Many epidemiological studies have been elucidated association between both diseases. Besides malnutrition, HIV, smoking and alcoholism DM have been recognized recently as a risk factor for TB. The biological support of causal relationship has been evident between DM and host immunity of TB. The situation changed dramatically in the last decade when the recognition of DM accepted as an unfolding epidemic in low and middle income countries [1]. A study conducted in Congo showed that TB patients were more likely to increased rate of TB treatment failure, defaults and relapses, deaths, delayed smear and culture conversions [2]. The World Health Organization in 2007 carried out a systematic review that also showed a strong links in both diseases.

Harvard University published a systematic review of all previous TB-DM co-infection related studies in 2008 also quantified the quadruple risk. A world health survey of 46 mainly low to middle income countries with high TB burden and DM prevalence reporting showed DM diagnosed patients were more likely to had symptoms of TB [3]. A systematic review of observational studies from 1980 to 2010 highlighted an increased risk of relapse and death of diabetic patients with TB Baker et al. 2011. A prospective cohort study which was nested in a nutritional trial in Tanzania investigated the diabetes role with a follow up period.

Their linear mixed-effect model showed delayed recovery of hemoglobin and body mass which are needed for functional recovery [4]. Asia is an epicenter of the larger growing burden of DM. An experimental study revealed this association with reduced morphage functions e.g. bactericidal actions, chemotaxis, phagocytosis and impairs the function of T-helper 1 cells their production of cytokines with proliferation [5]. Additional systematic reviews along with all land mark studies clearly showed that DM makes more than double high risk to develop TB. Both diseases deadly interaction constitutes public health threat worldwide [6].

Nevertheless, aggregated point to a significant impact of coexistence of TB-Diabetes is sensational. Thus, this issue requires an urgent attention. The renewal of old acquaintances constitutes a global threat, re-discovering due to high prevalence of type 2 DM that is now accounting for more than $90 \%$ of all DM cases. A similar situation of co-epidemic was also seen in the case of TB-HIV. There were enough evidences of TB-HIV emergence but delayed focus attention resulted in high rate mortality. Swaziland an African country where people affected by both disease, average life expectancy was at once cut in half within a decade. NowTB-DM co-infection is escalating in low and middle income countries [7]. As for now, unfortunately it is estimated that there are many more people in the world are living with TB-DM as compare living with TB-HIV. One third of the World population harbor TB germ making them more vulnerable to develop active TB if they face DM.

In Oct 2014, The WHO identified and raised an alarm for China, India, Pakistan, Brazil and Russia as these countries are facing DM epidemic with highly populated nations adding risk of high contagion. Now South Asia is facing a looming co-epidemic of TB-DM. India with some high burden states estimated 20 percent of active TB cases are found among diabetics. Neighboring China is also facing same situation [8]. In Pakistan the strong evidences of deleterious effect of one disease to another has potential impact that can be observed in areas where TB and DM are more prevalent. Pakistan is facing challenges in controlling dual diseases with increasing high rates of DM. In 2014 there were approximately 7.1 million diabetic patients that will increase 11.4 million in 2030. Unfortunately, 88,000 people die annually due to DM out of which 35,615 men and 52,397 women. Among top ten countries Pakistan ranks 7 th with highest prevalence of DM with fastest increasing in number. Pakistan ranks 5th worldwide on the other hand in high TB prevalent country with 420,000 new cases annually [9].

DM and TB interact with each other on a number of different levels that each disease exacerbating to other. DM triples a person's risk of developing TB [10] The likelihood that a person with TB will die, or will get TB again after a successful treatment, is also significantly higher among people with DM Baker et al. [11]. The research also shows that people who are being treated for TB, those with DM remains contagious longer than those who do not have DM. Diabetic patients are at a greater risk to contract TB while DM worsens the course of TB. TB makes worsen glycemic control in diabetic patients. TB patients who got DM have poorer treatment outcome facing $69 \%$ increase in treatment failure and increase fourfold the risk of post treatment relapse [11]. DM may make less effective TB frontline drug RIFAMPICIN that complicate DM management by worsening glycemic control. DM is associated with less rifampicin concentration resulting a drugdrug interaction in oral hypoglycemic drugs and anti-TB drug.

The hepatic toxicity of TB drugs is a risk factor associated with DM. Thus TB drugs lower concentration with increased hepatic toxicity leads to disease recurrence and high death rate respectively [12]. Rifampicin is an inducer of metabolizing enzymes in liver that decreases pharmacokinetic parameters of oral diabetic agents. In turn this impairs glucose level in coinfected patients. Cumulative evidences suggest to pay more attention to treat TB patients with DM. Now DM is a well-known risk factor that is facilitating and accelerating the conversion of latent TB to active form as well reactive the old TB disease. 
Several reports showed higher prevalence of DM in TB patient then in general population from all over the world. Co-epidemic growing trend refers to perfect storm which could reverse the progress that made against TB over last decades. Link of coinfection established in dual ways is an open challenge to global TB control program.

\section{Objective of the Present Study}

The health experts warn that TB spread to new heights affecting diabetic and non-diabetics in unprecedented numbers. People with Diabetes are more susceptible to catch the TB bacteria as their immune system usually compromised due to metabolic disorder. There is no research work done to assess the prevalence of DM in TB patients yet in this area as per to our knowledge. Thus the aim and objective of this study was to determine the prevalence and magnitude of DM with associated risk factors in patients with active TB at a tertiary care hospital in Rawalpindi District in the Punjab province of Pakistan.

\section{Conceptual Framework}

TB and DM are interrelated with each other on a number of different demographic levels. For instance, age, gender, family history, education, locality, marital status and socio-economic status do effects on both diseases. Smoking leads an individual susceptible to TB because there is a strong link and association between tobacco and TB. Smoking damages lungs and harm body's immune system. In result smokers cannot combat TB infection; moreover, smoking reduces TB treatment effectiveness which leads longer period infection eventually more severe form of disease. Certain occupational exposures excess the risk of developing TB. Then marital status found to be associated with TB. Spouses are more susceptible to develop active TB. While age is linked with both diseases as increasing age is actually advancing towards type 2 diabetes and low immunity results in active TB. In Locality the rural dwellers are more prone to develop TB while urban dwellers are more susceptible to develop type 2 diabetes due to sedentary life style and highly dense food intake. Socio-economic status has same impact like locality. High socioclass has more Diabetes while low socio-class is more prone to develop TB. Family history associated with dual diseases. TB and Diabetes impact on each other in fueling co-epidemic (Figure 1).

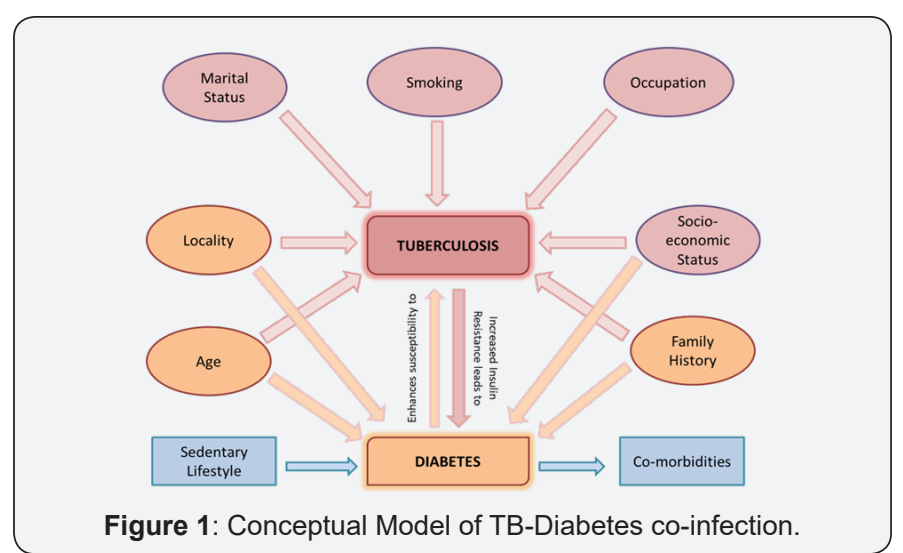

\section{Methods}

A cross-sectional hospital-based study was employed to assess the prevalence of DM and associated risk factors in TB patients from March 2016 to June 2016. The participants of the study were recruited from Government TB that is situated in Punjab province of Pakistan. The study confirmed the Helsinki Declaration and the formal permission was taken from institutional review board of Quaid-e-Azam University as well from the authorities of TB hospital to conduct this study. The confidentiality and anonymity of collected information was maintained through using the code numbers for study participants. All consecutive subjects with active pulmonary and extra-pulmonary TB who volunteered to participate and signed a written consent were enrolled. The study participants were given the informed consent prior to enrolment in the study. Consent forms were provided in English and Urdu language. All information about the research was given to participants 'prior the study than written consent was obtained. Study participants were assured about confidentiality, anonymity of the data and protection.

All study participants had a right to quit at any stage during the study. Thus 248 consecutive patients with physician diagnosed TB (both pulmonary and extra pulmonary) who consented were recruited into the study. The sample size was determined according to the World Health Organization and International Diabetes Federation Report, which is $16 \%$ prevalence of DM in TB patients in Pakistan (WDF \& IUATLD, 2014). For sample size calculation Open Epi software was used. The estimated population was utilized one million with confidence interval $95 \%$ and $5 \%$ level of significance. By using $20 \%$ of non-response rate to compensate missing data, the sample size was raised to $248(207+20 \%)$ to increase the statistical power of the sample. Systematic sampling with a random start technique was employed for this study. The TB hospital's OPD is open six days a week. Daily average number of diagnosed TB patients was approximately 40 . Total working days for out-patient department in a month were 25 days, so total population of diagnosed TB patients in TB hospital per month was $40 \times 25=1000$. While using systematic sampling formula (Sampling interval $=$ total population $\div$ sample population) " $1000 / 248=4.03^{\prime}$ every $5^{\text {th }}$ patient was recruited in the study.

A pretested structured questionnaire was administered after a pilot study. Modifications were made in the questionnaire after a pilot study. OPD slips of TB patients were collected from nursing counter; those patients who met the inclusion criteria were listed. In case of refusal the next patient in turn was considered. The patients were interviewed to sought information on dual disease status. A well trained investigator recorded the anthropometric measurements viz. (BMI) height, weight, and waist circumference by standard procedure. The (BMI) quantify the amount of tissue mass (bone, muscle and fat) in an individual and categorize that person as underweight, normal weight and obese based on that value. 
By using formula

$$
\mathrm{BMI}=\frac{\text { mass }_{\mathrm{kg}}}{\text { height }_{\mathrm{m}}^{2}}=\frac{\text { mass }_{\mathrm{b}}}{\text { height }_{\text {in }}^{2}} \times 703
$$

The TB patients were defined as underweight (BMI $<18.00$ $\mathrm{kg} / \mathrm{m} 2$ ), normal (BMI $\geq 18.00$ and $<25.00 \mathrm{~kg} / \mathrm{m} 2$,), overweight and obese (BMI $\geq 25.0 \mathrm{~kg} / \mathrm{m} 2$ ). The waist circumference (WC) was measured at the midpoint between lower boarder of rib cage and the iliac crest. The two blood pressure measurements were taken using the sphygmomanometer with the patient in sitting posture, and the average of two readings were recorded respectively. DM was confirmed by determining randomly any value between $140 \mathrm{mg} / \mathrm{dl}$ to $199 \mathrm{mg} / \mathrm{dl}$ for pre-diabetes and for the Diabetes diagnosis, any random blood glucose that was greater than $200 \mathrm{mg} / \mathrm{dl}$. The whole data was cleaned, entered and analyzed using the software statistical package for social sciences (SPSS) version 21.

\section{Results}

Among 248 TB patients 128 (51.6\%) were males and 120 $(48.4 \%)$ were females and the mean age of the participants were $42.29 \pm 18.22$ years. The participant's age range was between 18 to 79 years. The mean age of TB patients who had Diabetes was $41.5 \pm 13.9$ years. DM was found in $52(21 \%)$ patients. Among them there were 20 males and 32 females. There were 14 newly detected DM patients while 38 were known cases. In univariate logistic regression age, area of residence, family history of TB, knowledge about diabetes, relapse and co-morbidities were found more likely to associated at $\mathrm{p}<0.20$

Age was 3.5 times found to be more likely to associated as [OR 3.5(95\% CI=1.839-6.663)]. Rural area was found 2.2 times more highly effected as [OR $2.2(95 \% \mathrm{CI}=1.162-4.164)]$. Family history of TB was 2.7 times more likely to be associated with TB-Diabetes as [OR 2.7(95\% CI=1.447-5.186)]. Knowledge of diabetes was found 19 times more likely to be associated with dual disease as [OR 19.8(95\% CI=9.325-4.422)]. Family history of diabetes was 13 times more likely to be associated with TBdiabetes as [OR 13(95\% CI=6.406-26.642)]. Relapse was 14 times found more likely to be associated with dual disease as [OR 14(95\% CI=6.839-30.145)]. While the co-morbidities were 19 times more likely to be associated with TB-diabetes as [OR $19(95 \%$ CI=8.232-47.050)].

Logistic regression was done to assess the impact of different factors on the Diabetes Mellitus among TB patients. A value of $\mathrm{P}<0.05$ was considered statistically significant. Multivariate model contained five independent variables (age, family history of Diabetes, Diabetes knowledge, TB-relapse and co-morbidities). Model containing all predictor variables was statistically significant, $\chi 2(5, \mathrm{~N}=248)=88.97, \mathrm{p}<.001$, indicated that the model was able to distinguish between different predictors.

The model explained as a whole between 33.5\% (Cox and Snell R square) and $57.8 \%$ (Nagelkerke R squared) of the variance in Diabetic status with correctly classified $84.4 \%$ of all cases. Five independent variables made a unique statistically significant contribution to the model; those were age group, Diabetic family history, Diabetes knowledge and TB-relapse and co-morbidities. Among them the strongest predictors were comorbidities and Diabetes knowledge with odd ratio 8.22 and 7.1 respectively which indicated that both these variables were over 7 times more likely to report controlling for all other factors in our model.

\section{Discussion}

Through this institutional based cross sectional research our findings are in line with reports of high prevalence of DM in TB patients with different region of the world. For instance, Mexico (25\%), Tanzania (16.7\%), Indonesia (13.2\%), and Africa (44\%) having wider range of dual epidemic [13]. Studies conducted for dual disease burden in Asian region had reported prevalence ranging between $14-40 \%$. The prevalence of DM among active TB patients $7.28 \%$ was higher compared to the published prevalence of DM in the general population. A cross- sectional study in Lahore, Pakistan concludes 5.69\% newly diagnosed Diabetes among TB patients during the course of investigation [14]. In our study DM found higher among pulmonary TB patients rather than extra pulmonary TB patients as reported in an Indian study [15]. Similar finding was concluded by Hong Kong study Leung et al. [16]. In Indonesia, where confirmed prevalence of Diabetes in TB patients was $14.8 \%$ compared with $3.2 \%$ in general population found Diabetes strongly associated with pulmonary-TB [16].

In a retrospective study by India found DM proved a negative effect on immune response and enhanced TB morbidity [15]. A state wide cross sectional study concluded that half of the TB patients had Diabetes [13]. Zambia is another country with high TB burden has evaluated in a prospective study that TB is significantly associated with DM Bates et al. [16]. Another land mark systematic review in2014 results showed that both diseases interaction constitutes worldwide health threat [17]. Among study participants' malnutrition was more common as (median BMI 17.7 vs. $21.5 \mathrm{~kg} / \mathrm{m} 2$ ). Women were found more vulnerable and undernourished in our study (median BMI 17.7 vs. $21.5 \mathrm{~kg} / \mathrm{m} 2$ ). A cross sectional study conducted in Nigeria also found undernourished and weight loss the predominant symptoms in most of the patients [18]. Moreover, majority of the males were educated and employed as compare to women. Gender difference had been seen clearly in educational status.

There were 109 (44\%) TB patients who belonged to 18 to 35 years' age group. This shows that their productive time of life period is compromised that is very unfortunate for them. Pakistan is a developing country with majority young population, thus TB as comorbidty with DM cannot be ignored. The majority of Diabetic patients were in their middle ages with (36-79) years similar to other studies. Like Indian study where these patients were above age 40 [19]. The dual disease association 
is well documented with substantial evidence to support the fact. We found higher prevalence of DM among women than men that could be more age of women as men were in their younger ages since increased age emerged a significant risk factor for DM. Conversely, it may also possible that TB has induced glucose intolerance and was more prevalent among women than men. Poor glycemic control with lack of non-specific antibody production can be attributed with adaptive immune mechanism which may facilitating factors of past infections resurgence.

The present study also identified a variable that has a most important influence on occurrence of DM in TB patients. Those patients who have family history of DM got co-infection. Most of the TB patients were married and belonged to joint families. The possible reason of this could be crowding living conditions. Moreover, who were living with a TB patient in family identified as an important factor for occurrence of TB which infect might be frequent contacts in household lead to increased transmission. Moreover, common risk factors of DM with positive family history were seen in the current study as well from Indian study [20]. In addition, early diagnosis and treatment of DM in low resource setting is difficult which probably increase further proportion of diabetics developing TB.The preponderance of DM in urban TB patients was not seen in present study as most of the patients belonged to rural area. A study conducted to assess difference in urban and rural area for DM with TB co-infection found urban residence significantly associated [21]. The majority of known Diabetic cases were on regular treatment of DM. Relapse was significantly associated with DM despite the glycemic control treatment. In Thailand, a prospective study found relapse significantly associated with DM [22].

DM knowledge has been well acknowledged tool for prevention and management of DM. The management of this chronic disease requires rational therapeutic approaches with proper education and counseling from health care providers. The individuals 'self-care practices are highly influenced by their knowledge and attitudes. The more knowledge about DM the more self-management skills can improve. We found the level of awareness very low in Diabetic patients. The DM awareness studies in Pakistan found same results along with myths and strong beliefs regarding diet and insulin complications. Moreover, absence of formal education makes the situation more shocking and adverse.

Sedentary occupational status and smoking was more prevalent in males put them higher risk of co-infection. Changing life style with less physical activity and more junk food have contributed the disease on rise in Pakistan both in urban and rural areas. Many patients were reluctant to answer about alcohol use due to stigmatized issue which attributed to social desirability bias. Few patients had hypertension and abdominal obesity. Similar finding can be found in an Indonesian study where Diabetes was strongly associated with TB in non-obese and young study subjects [23].

\section{Strength and Limitation of the Study}

The strength of this study is that there was no study done before in this area (District Rawalpindi) to assess the magnitude of Diabetes Mellitus in TB patients. Since our study is hospital based it may be biased. This cross-sectional study therefore may not truly represent the true condition of TB-Diabetes coinfection in the community. Due to limited time and resources the results were confined and further a prospective study with large population of Diabetics and non-diabetics with TB is needed for determining validity of results. Nonetheless, Diabetes diagnosis was based on one point in time glucose monitoring rather repeated measurements to diagnosis confirmation which is common in virtually all epidemiological studies of Diabetes. In result significant misclassification of Diabetes cases due to mixture of biological variation in glucose levels of blood and measurement error. It led likely to underestimation of TBDiabetes association.

In general, this study with other two studies in Pakistan so far according to our knowledge showed high burden of Diabetes among TB patients. Therefore, bi-directional screening is inevitably relevant especially in TB endemic countries like Pakistan where DM is soaring.

\section{Conclusion}

The baseline data before this study was limited from Rawalpindi District. Our study highlighted that DM is $21 \%$ present among TB patients which is higher than expected. The study revealed that 14 (7.28\%) Diabetics were newly diagnosed that raises concerns of routine screening for DM in TB patients is essential. The mean age of dual disease patients indicates that at productive age when they need to earn to support their families, unfortunately they have to face economic burden of health expenditures. The present epidemiological study indicates that rapid increase of Diabetes with $\mathrm{TB}$, yet a clinical importance and potential public health threat seems to be ignored largely. Irrespective of its causal or co-morbid factors good quality implementation is urgently needed for robust action plans to address this dual burden in Pakistan. Hence TB patient's glucose levels should be timely assessed and monitoring along with smoking cessation should be a main stay in ceasing the progression of co-infection.

\section{Acknowledgment}

We thank to all the individuals for their cooperation and participation in the study.

\section{Conflict of Interest}

This study has no conflict of interest to declare by any author.

\section{References}

1. Jeon CY, Murray MB (2008) Correction: Diabetes Mellitus Increases the Risk of Active Tuberculosis: A Systematic Review of 13 Observational Studies. PLoS medicine 5(8): e152. 
2. Goldhaber-Fiebert JD, Jeon CY, Cohen T, Murray MB (2011) Diabetes mellitus and tuberculosis in countries with high tuberculosis burdens: individual risks and social determinants. Int J Epidemiol 40(2): 417 428

3. Goldhaber-Fiebert JD, Jeon CY, Cohen T, Murray MB (2011) Diabetes mellitus and tuberculosis in countries with high tuberculosis burdens: individual risks and social determinants. Int J Epidemiol 40(2): 1-12.

4. Faurholt-Jepsen D, Range N, Praygod G, Kidola J, Faurholt-Jepsen M, et al. (2012) The Role of Diabetes Co-morbidity for Tuberculosis Treatment Outcomes. A Prospective Cohort Study From Mwanza, Tanzania. BMC Infect Dis 12: 165

5. Damtew E, Ali I, Meressa D (2014) Prevalence of Diabetes Mellitus among Active Pulmonary Tuberculosis Patients at St. Peter Specialized Hospital, Addis Ababa, Ethiopia. World Journal of Medical Sciences 11(3): 389-396.

6. Badawi A, Sayegh S, Sallam M, Sadoun M, Al-Thani M, et al. (2014) The Global Relationship between the Prevalence of Diabetes Mellitus and Incidence of Tuberculosis: 2000-2012. Glob J Health Sci 7(2): 183-191.

7. Stevenson CR, Forouhi NG, Roglic G, Williams BG, Lauer JA, et al. (2007) Diabetes and tuberculosis: The impact of the diabetes epidemic on tuberculosis incidence. BMC Public Health 7(1): 234

8. Ruslami R, Nijland HM, Adhiarta IG, Kariadi SH, Alisjahbana B, et al (2010) Pharmacokinetics of Antituberculosis Drugs in Pulmonary Tuberculosis Patients with Type 2 Diabetes. Antimicrob Agents Chemother 54(3): 1068-1074.

9. World Health Organization. WHO Global TB Report 2013. Geneva: WHO office.

10. Lönnroth K, Roglic G, Harries AD (2014) Improving tuberculosis prevention and care through addressing the global diabetes epidemic: from evidence to policy and practice. The Lancet Diabetes \& Endocrinology 2(9): 730-739.

11. Faurholt-Jepsen D, Range N, Praygod G, Kidola J, Faurholt-Jepsen M, et al. (2012) The Role of Diabetes Co-morbidity for Tuberculosis Treatment Outcomes. A Prospective Cohort Study from Mwanza, Tanzania. BMC Infect Dis 12: 165

12. Damtew E, Ali I, Meressa D (2014) Prevalence of Diabetes Mellitus among Active Pulmonary Tuberculosis Patients at St. Peter Specialized Hospital, Addis Ababa, Ethiopia. World Journal of Medical Sciences 11(3): 389-396.
13. Balakrishnan S, Vijayan S, Nair S, Subramoniapillai J, Mrithyunjayan S, et al. (2012) High Diabetes Prevalence among Tuberculosis Cases in Kerala, India. PLOS One 7(10): e46502.

14. Usmani RA, Nasir MI, Wazir S, Pervaiz Z, Zahra T, et al. (2014) Diabetes mellitus among tuberculosis patients in a tertiary care hospital of Lahore. J Ayub Med Coll Abbottabad 26(1): 61-63.

15. Viswanathan V, Kumpatla S, Aravindalochanan V, Rajan R, Chinnasamy C, et al. (2012) Prevalence of diabetes and pre-diabetes and associated risk factors among tuberculosis patients in India. PloS one 7(7): e41367.

16. Alisjahbana B, Sahiratmadja E, Nelwan EJ, Purwa AM, Ahmad Y, et al. (2007) The effect of type 2 diabetes mellitus on the presentation and treatment response of pulmonary tuberculosis. Clin Infect Dis 45(4): 428-435.

17. Riza AL, Pearson F, Ugarte-Gil C, Alisjahbana B, van de Vijver S, et al. (2014) Clinical management of concurrent diabetes and tuberculosis and the implications for patient services. Lancet Diabetes Endocrinol 2(9): 740-753.

18. Olayinka AO, Anthonia O, Yetunde K (2013) Prevalence of diabetes mellitus in persons with tuberculosis in a tertiary health centre in Lagos, Nigeria. Indian J Endocrinol Metab 17(3): 486-489.

19. Viswanathan V, Kumpatla S, Aravindalochanan V, Rajan R, Chinnasamy C, et al. (2012) Prevalence of diabetes and pre-diabetes and associated risk factors among tuberculosis patients in India. PloS one 7(7): e41367.

20. Shetty N, Shemko M, Vaz M, D’souza G (2006) An epidemiological evaluation of risk factors for tuberculosis in South India: a matched case control study. Int J Tuberc Lung Dis 10(1): 80-86.

21. Stevenson CR, Forouhi NG, Roglic G, Williams BG, Lauer JA, et al. (2007) DM and tuberculosis: the impact of the DM epidemic on tuberculosis incidence. BMC Public Health 7: 234

22. Duangrithi D, Thanachartwet V, Desakorn V, Jitruckthai $P$, Phojanamongkolkij K, et al. (2013) Impact of diabetes mellitus on clinical parameters and treatment outcomes of newly diagnosed pulmonary tuberculosis patients in Thailand. Int J Clin Pract 67(11) 1199-1209.

23. Alisjahbana B, van Crevel R, Sahiratmadja E, den Heijer M, Maya A, e al. (2006) Diabetes mellitus is strongly associated with tuberculosis in Indonesia. Int J Tuberc Lung Dis 10(6): 696-700.

\section{Your next submission with Juniper Publishers} will reach you the below assets

- Quality Editorial service

- Swift Peer Review

- Reprints availability

- E-prints Service

- Manuscript Podcast for convenient understanding

- Global attainment for your research

- Manuscript accessibility in different formats ( Pdf, E-pub, Full Text, Audio)

- Unceasing customer service

Track the below URL for one-step submission https://juniperpublishers.com/online-submission.php 